\title{
The reactive sputtering of oxides and nitrides
}

\author{
R P Howson
}

Dept of Physics, Loughborough University of Technology, Loughborough, Leics., UK. LE11 3TU.

Abstract: Recent developments in the techniques used to produce surface layers of oxides and nitrides by reactive sputtering are considered. These techniques have to give films which can be produced onto large-area, low-temperature substrate materials, such as glass and polymer. It is shown that this has led to the adoption of ion-assisted processes. In particular the use of plasmas leaked from magnetron sputtering sources is shown to have the ability to create the intense low-energy bombardment that has been found to be most beneficial in forming the film structures that are required.

Examples of the use of reactive unbalanced magnetron sputtering for the preparation of oxides and nitrides of silicon, titanium, aluminium etc. is given with discussion of their application to provide surface coatings having desirable properties.

\section{INTRODUCTION}

Modern technology requires coatings to modify the behaviour of the surface of a material, which in general has a relatively large area, and for economic reasons is made of an easily available, low-temperature material such as mild steel, glass or a polymer. The coatings that are required are refractory in character and they have to be deposited onto substrates which cannot tolerate high temperatures. Vacuum deposition allows the preparation of such materials in a relatively simple way, particularly the process of reactive sputtering of oxides and nitrides, where the metal is sputtered in a residual atmosphere containing the gaseous component. The appearance of low-pressure sputtering processes has demonstrated the advantages obtained with a higher energy depositing species; the films are generally more adhesive with a dense structure and reactions with the reactive gas, present in the residual atmosphere, provides compound film growth more easily. The outstanding advantages of a vacuum process are then seen to lie in the ability to use ions, which can be provided with much more energy than can be achieved by simply raising the temperature.

It is necessary that the pressure of the residual atmosphere is sufficiently low to allow a mean-free-path of sufficient length, so that the high energy acquired is not dissipated in gas-phase collisions. This energy can be directed to the surface of a growing-thin-film to give actuation of adhesion, reaction, cleaning and structural effects. One technique which has proved outstandingly successful in this regard is that of magnetron sputtering, where a magnetic field is used to confine the gas discharge plasma close to the cathode target. In the reactive-sputtering technique material is transported to a growing film surface, through the vacuum, after being vaporised by being ejected from a cold target through a collision with a high energy inert gas ion. It acquires sufficient energy to initiate reactions on the growing film surface with the oxygen or nitrogen supplied by the residual atmosphere. 
Unfortunately this simple concept is not quite as easy as it at first appears. The sputtering cathode will also be subject to bombardment with the reactive gas component of the residual atmosphere which will cause reactions to occur on it $s$ surface, and compound materials to be formed which can have very different properties from those of the original cathode material. In particular the material could be an insulator and have a very different sputtering coefficient. This phenomena is called target poisoning.

It is the simple solutions to the problems of target poisoning and ion-bombardment of the growing film which are provided by the use of the unbalanced magnetron that are the basis of this paper.

\section{ION-ASSISTED PROCESSES}

The requirements of an ion assisted process are very clear: A means of getting energetic ions to the surface must be provided which does not interfere with the material transfer process. The ions that are used can be those of the material being deposited or those of an inert gas.

Techniques have evolved through involuntary use of ion energy, with the use of low pressure sputtering, where some of the energy used to sputter the material is given to the sputtered atoms and is not dissipated by gas phase collisions. Outstanding amongst these techniques has been the application of radio frequency power and the use of magnetic confinement in devices such as the planar magnetron. These techniques allow the use of low gas pressures, which give mean free paths for collision between a sputtered particle and the residual gas which is longer than the source to substrate distance, allowing some of the high energy used for the process to be transfered to the growing film. RF processes have the other advantage that all surfaces in such a process are active electrodes and will be bombarded with ions whether insulating or not. In fact this bombardment cannot be avoided.

The types of vacuum systems that can be used for the economic production of the large areas means that processes tend to be "dirty", with the contamination introduced from the residual atmosphere of the vacuum system being high. The surface preparation and cleaning provided by scrubbing the growing film surface with inert-gas-ions removes poorly adhered contaminents and poorly integrated primary material. This means that much higher quality films can be produced than might be expected. The effects that might be expected are shown in Fig. 1, which is taken from Schiller [1]. The high rate that is required of the process for economic reasons is also a requirement for creating purer films. If the background pressure is high, and results in a high rate of delivery of impurity to the surface of the growing film, then the higher the rate of delivery of the required material the purer the film, that is obtained, will be. Also, because of the large amount of material being condensed on surfaces adjacent to the cathode, a getter effect of condensable impurity is obtained; the contaminant present in the residual atmosphere is removed faster than it can be replaced, especially as nearby desorbing surfaces will be now covered with freshly condensed material. This effect can be remarkably effective in creating pure films in poorly pumped systems [2].

Ions can, of course, be generated in a separate process and accelerated onto the growing film surface to give energy to the depositing material. It is however difficult to achieve deposition rates which are sufficiently high to overcome contamination effects, to provide bombardment of sufficiently low ion energy, so as to avoid radiation-type damage to the structure of the film, and to give economical rates [3].

In recent times techniques of providing relatively low-voltage-ion bombardment of a growing film have appeared which have been shown to result in improved properties of the 


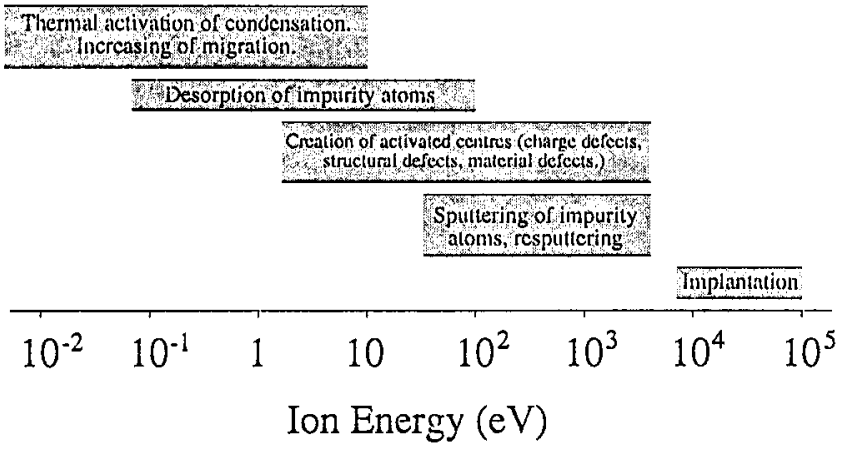

Figure 1.The effects that are seen on growing film surfaces as a function of the energy of the ions that are incident on it .

films that are made. They involve placing the insulating or isolated substrate in a dense DC plasma, the self-bias that appears on the surface due to the need for electron and ion currents to equalize causes the ions to be accelerated through voltages of up to 100 volts [4]. This dense plasma is created by passing a large current of electrons through a lowpressure inert gas. This current is in the region of $200 \mathrm{~A}$, at about $80 \mathrm{~V}$, which is close to the optimum required for ionization of the Argon gas that is used. The pressure required is below that which would cause significant collisions of evaporating material with the constituents of the residual atmosphere.

\section{UNBALANCED MAGNETRON}

In recent times it has been demonstrated that the field localising the plasm close to the cathode in a planar magnetron can be unbalanced to allow some of this plasma to be incident on the growing film surface [5]. If this surface is isolated it will acquire a bias potential of the order of $30 \mathrm{~V}$ and subject the film to ion bombardment of that energy [6]. This is the range identified as having the most potential for improving the properties of deposited films. The unbalancing of this field can be easily built into the system or controlled with a solenoid behind either the substrate or target. When even numbers of multiple units are used it has been shown that alternating the pole positions in the individual magnetron units leads to a closed field condition which traps the leaking plasma in the volume of space between them. This trapped plasma can be used to provide ions for bombardment of the growing film. This technique has proved very effective in providing high quality thick films of titanium/alumium nitrides and carbides especially when used with PEM control [6].

\section{REACTIVE SPUTTERING}

One of the effects of ion bombardment is to affect the reaction processes, occuring in reactive sputtering, and it is necessary to consider this technique. Commonly the admission of the reactive gas in a reactive sputtering process is done by control of the flow, with a simple mechanical valve or an electronic feedback device which can indicate the magnitude of that flow. In high rate reactive sputtering with a planar magnetron consumption of the reactive gas by the process is greater than that being pumped by the system and conditions are often created where the process is unstable, switching between "metallic" and "oxide" sputtering modes without allowing access to the intermediate point, which is the one generally required [1]. The sputtering of an oxide covered target is generally not desirable because the sputtering rate of the oxide is much lower than that of 
the metal and the appearance of an insulating surface on the cathode causes arcs if dc is used, and rf power is required. This effect is particularly difficult to solve for the oxides of alumium and titanium, materials in great demand as thin film elements, because the rate of deposition obtained by sputtering the oxide is unacceptibly low. Though the oxides of materials such as indium, zinc and tin do not give such a low rate and can be sputtered from an oxiding metal target with dc, without arcing, and have been used for coating large areas of architectural glass [7]. The use of very high speed pumping systems has proved to provide a solution to the problem of the reactive sputtering of a metal titanium target to provide a reasonable rate for the preparation of large areas of the oxide [8]. The pumping speed of a system does affect the process of the reactive sputtering of indium-tin oxide [9]. The simple solution requires large pumps, with arrangements of the system to give high gas conductances, which can be difficult to arrange and is expensive. A solution to the instability can be obtained by providing control of the partial pressure of the reactive gas with rapid feedback. Following Chapin and Schiller [10] we have found that this can best be done by using an observation of the spectral line emission of the gas or the sputtering metal to control the admission of the reactive gas [11].

The object of the process is to create films of closely controlled stoichiometry and structure. The rate of incidence of the sputtering metal and the consumption of the reactive gas from the residual atmosphere, together with the reaction kinetics of the surface reaction will obviously be important in this and these can be measured through the power provided to the magnetron and the amount of reactive gas admitted in excess of that which flows into the pumps $[12,13]$ The energetic state of the reactants will be an important factor in these kinetics and these will be determined by the ionisation state of the incident particles and the potential through which they have been accelerated, particles which have been neutrallized after acceleration can also be significant in this.

An alternative to the simultaneous supply of reactants to the growing film surface is to supply the materials in sequence, with the layers that are formed being of monolayer dimensions, to allow easy access of the materials to each other [14]. Such a process has proved to be very effective in certain circumstances. Such a process has been called MAGIC by Martin [15]: MAGnetron sputtering with Ion Conversion. We have used a dense plasma to provide the activation of the reactive gas in a rotating disc system; we have called this SPECIAL MAGIC: Sequential Plasma Enhanced Conversion In A Layer created

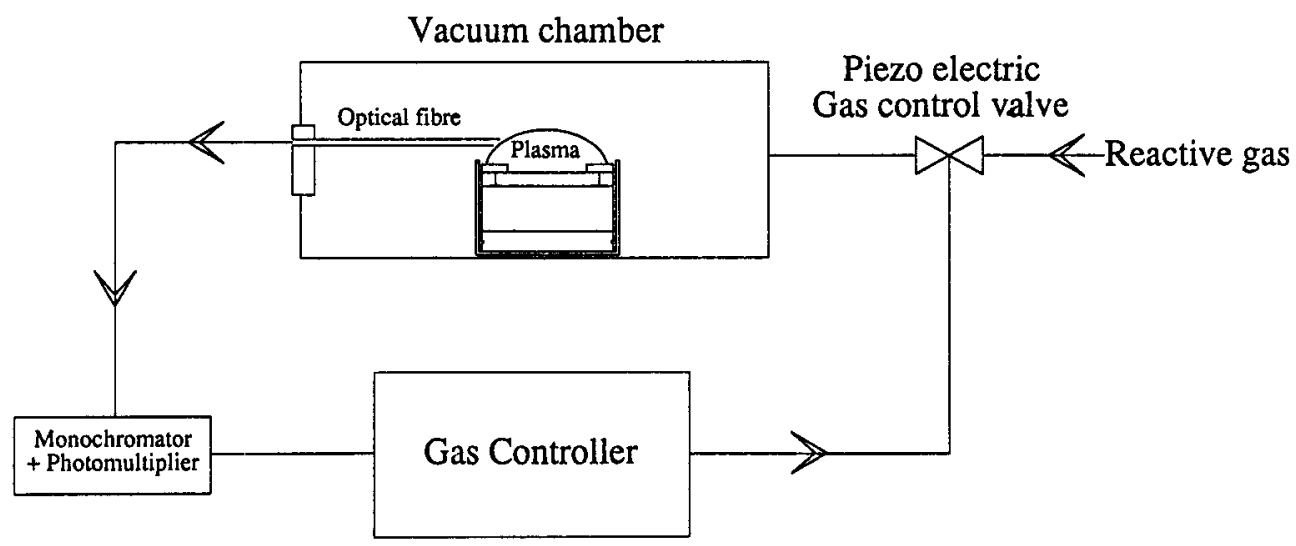

Figure 2. Plasma Emission Monitoring (PEM) used to control reactive sputtering with a planar magnetron. 


\section{SCEEVATIC DIAGRAI OR TBR COATING PROCESS}

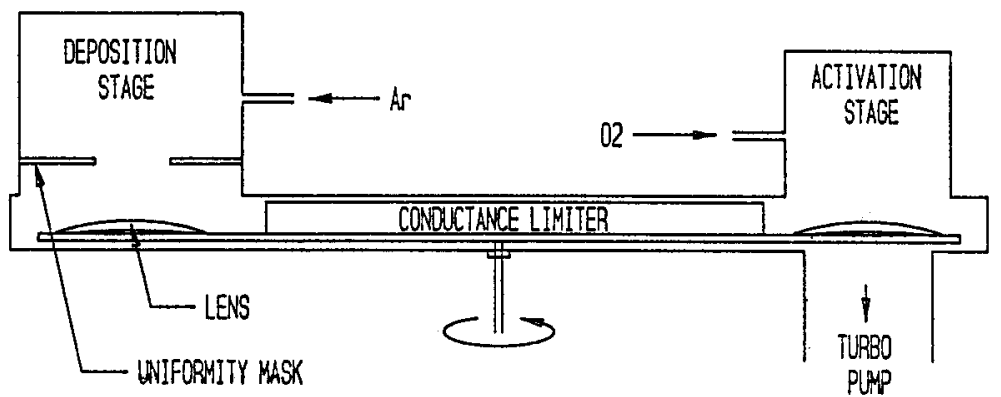

Figure 3. The SPECIAL MAGIC process for the sequential anodisation of a metal film.

by MAGnetrons In Cans. This process forms the basis of a precision coating machine for the coating of ophthalmic lenses which is completely stable and can operate with a minimum of control systems [16] and is shown in Fig. 3. It is used by OCLI in their METAMODE process where metal layers produced by sputtering from non-oxided targets are oxidised with an ion-gun [17]. This is achieved on a rapidly rotating cylinder to give the rates that are required for commercial operation.

\section{EXAMPLES}

Some examples of the use of ion-assisted processes to give high quality coatings of oxides and nitrides will be considered:

\section{Titanium Compounds}

Titanium oxide films have been grown in a very thin film form, suitable for application as optical transmission filters with the use of an unbalanced magnetron [18].

The influence of the ion bombardment allows very thin films of TiN to be obtained with a low electrical resistivity, which is lower than that of titanium metal [12]. An interesting feature of this PEM controlled process is that observation of the flow of reactive gas into the system allows the properties of the resulting films to be predicted as is shown in Fig. 4 . Similar techniques using multiple magnetrons are emerging for the production of high performance wear resistant coatings. The conducting substrates are biased to add energy to the growing film by bombarding it with ions available with these processes [6].

\section{Silicon Oxide}

Silicon can be DC sputtered with an unbalanced magnetron onto unheated glass substrates in atmospheres of argon and reactive gas. The reactive gas can be a mixture of oxygen and nitrogen to give mixed oxide-nitride films with a range of refractive indices [19].

\section{Transparent Conducting Oxides}

A process for the reactive sputtering of indium-tin oxide using PEM control and observation of the oxygen gas flow has been established which gave transparent films with 


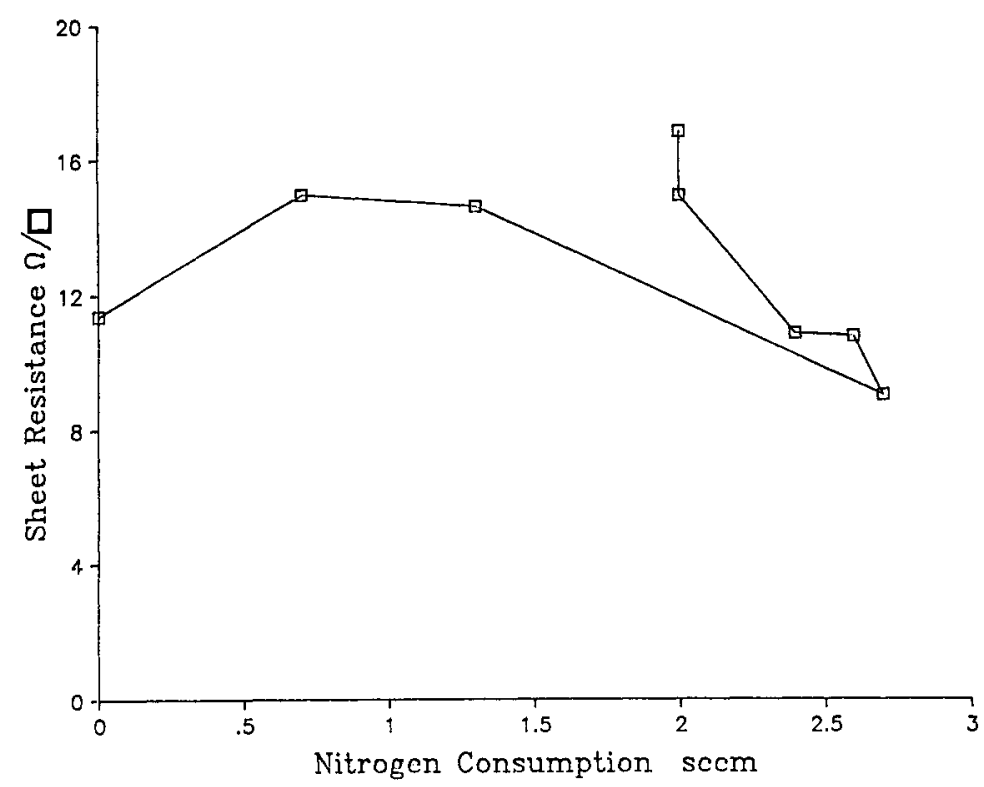

Fig. 4. The flow of nitrogen into a closed system, used for the reactive sputtering of titanium nitride, displayed as a function of the resistivity of the film that is produced. PEM is used to control the operating point.

a resistivity of $4^{*} 10^{-6} \mathrm{ohm}$-meters when made onto room temperature polymer substrates [20].

Indium-tin oxide is a useful sustem to consider when made by reactive sputtering; for the properties that are required of high electrical conductivity and visible transparency are easily observed and related to deposition parameters. Targets of metal alloys or compacted oxide can be used. Sputtering from the metal gives a higher rate but requires much greater precision in the control of the partial pressure of the oxygen that is required. The optimum alloy composition is a mystery in most systems. We have looked at this problem with a concentric dual metal source magnetron to vary the proportion of indium to tin and found that the best properties were obtained with pure indium. The mechanical properties of the films improved with alloying with tin but the electrical properties deteriated. The influence of ion bombardment from a plasma was interesting in that, as with rf [21], no improvement in the properties was obtained but suprisingly despite a move to lower partial pressures of oxygen for the preparation of optimum films no increase in sputtering rate was obtained, which would be expected with a move to a target less covered with oxide 'poison'. If material made at room temperature is annealed in vacuum or an inert atmosphere the resistivity falls to the value that is obtained by using that temperature in direct preparation. Resistivities down to below $2 * 10^{-6} \mathrm{ohm}-\mathrm{m}$ have been reported for films made on substrates approaching $400^{\circ} \mathrm{C}$. If annealing is undesirable or impossible our prefered technique is to sputter a metal target with PEM stabilization using the consumption of the reactive gas, above that going into the pumps, to provide an indication of the optimum preparation conditions. Such a process has proved sucessful with the much more difficult tin oxide system. The results [13] indicate how effective the ion-bombardment, obtained by deployment of the plasma from an unbalanced magnetron, can be on the reactivity of the process. 


\section{CONCLUSIONS}

In the preparation of optical films of carefully controlled properties on a large scale it can be demonstrated that considerable advantages can be brought to a process by the addition of energy at the surface from low-energy-ion bombardment, making the preparation of high quality films on temperature-sensitive substrates an economical reality.

Recent developments with unbalanced magnetrons has demonstrated that an isolated substrate can be bombarded with ions of the intensity and energy best suited to providing the densification of the film by immersing it in a low-pressure plasma. The pressure that this can be done is sufficiently low for a sufficiently long mean-free-path, for the depositing species, to be maintained. In a reactive process the activation provided by a plasma can result in the creation of denser films made at at lower partial pressures of the gas.

\section{ACKNOWLEDGEMENTS}

The author is pleased to acknowledge the work done by members of the Thin Film Group at Loughborough and in particular A G Spencer, M Stenlake and G Hall who performed much of the original research reported here.

\section{REFERENCES}

1. S. Schiller, U. Heisig and K. Goedicke, Proc.7th Intern. Cong. and 3rd Intern. Conf. Solid Surfaces, Vienna, (1977) 1545

2. H. C. Theuerer and Hauser J. J., J.Appl.Phys.,35, (1964) 554

3. J. Ebert, Surface and Coatings Technology, 43/44, (1990) 950.

4. H K Pulker, W Haag, M Buhler and E Moll, Proceedings of IPAT 85, Munich (CEP Consultants Ltd.), 299.

5. B. Window and N. Savvides, J.Vac.Sci.Technol., A4, (1990), 453

6. D. G. Teer, Surface and Coatings Tech., 39/40, (1989) 565

7. A D Grubb, T S Mosakowski and W G Overacker (Airco Temescal), Optical Thin Films, Proc.SPIE 325, (1982) 74.

8. Duratest Corporation, Private communication (1976).

9. R. P. Howson, and H. A. Ja'fer, J.Vac.Science and Tech., A10, (1985) 1774.

10. J. Chapin and C. R. Condon, 1979 US Patent 4, 166, 784.

S. Schiller, U. Heisig, G. Beister, K. Steinfelder, J. Strumpfel, Chs Korndorfer, and T.Seiber, Thin Solid Films, 118 (1984) 255.

11. R. P. Howson, A. G. Spencer, K Oka, and R.W. Lewin, J. Vac. Sci. Technol., A7, (1989) 1230.

12. R. Swady, H A Ja'fer and R P Howson, Vacuum, 297, (1993) 44.

13. R. P.Howson, H. Barankova and A. G. Spencer, Thin Solid Films, 196, (1991) 3.

14. S. Schiller, U. Heisig, K. Steinfelder, J Strumpel and W. Sieber, Vacuumtechnik, 30, (1981) 1 .

15. T. Martin, Presented at the AVS meeting Seattle 1991.

16. Applied Vision Ltd., Technical literature (1991).

17. J. P.Lehan, R. B. Sargent, and R. E. Klinger, J.Vac.Sci.Technol., A10, (1992) 3401.

18. R. P. Howson, ("Synthesis of Optical Thin Films: Fundamentals and Applications") in "Multicomponent and Multilayered Thin Films for Advanced Microtechnologies: Fundamentals and Applications", NATO/ASI Series E, O. Auciello and J. Englemann (Eds.), Kluwer Academic Publishers, The Netherlands (1993). 
19. R. P. Howson, G. Hall, and A. G. Spencer, Proceedings of IPAT 91, Brussels (CEP Consultants Ltd.), (1991) 128.

20. R. Lewin, R. P. Howson, C. A. Bishop, and M. I. Ridge, Vacuum 36, (1985) 95.

21. M. I. Ridge and R. P. Howson, Vacuum 34, (1985) 327. 